\title{
Research on Parameter Design of Multi - axis Hydrostatic Transmission Vehicle
}

\author{
Liang Zhao ${ }^{1,2, a}$, Jin Wang ${ }^{2}$ and Zheng-wu Zhang ${ }^{2}$ \\ ${ }^{1}$ Chang'an University, School of Construction Machinery, Xi'an ,China \\ ${ }^{2}$ Shaanxi University of Technology, School of Machinery Engineering, Hanzhong, China
}

\begin{abstract}
In order to obtain reasonable parameters in the design of driving system of multi-axis hydrostatic transmission vehicle, the working principle of single-side drive of hydrostatic transmission vehicle is introduced. The matching and control of engine and hydraulic pump are analyzed. According to the driving equation of vehicle, The driving force required for driving system is determined, and the parameters of hydraulic motor, hydraulic pump, system working pressure and braking system are designed and calculated, which provides the parameter design for driving system of multi-axis hydrostatic transmission Reliable theoretical basis.
\end{abstract}

\section{Introduce}

It is a special chassis for operation, such as concrete mixer truck chassis, concrete pump truck chassis and large fire engines, etc., must be a second type of chassis to be modified to meet the vehicle after the conversion requirements, such as full power Output force device, and even install the transfer case and vice engine. Hydraulic transmission technology with a wide range of speed, easy to control, can achieve stepless speed and other characteristics, can achieve the vehicle's stepless speed regulation and freestyle, in the construction machinery walking system has been a lot of applications ${ }^{1-2}$. As the need for special chassis models are growing, engineering machinery and often in a certain work area for microoperation. According to the characteristics of hydraulic transmission, the use of hydrostatic transmission of the way, so that the power transmission layout flexible and easy to achieve the vehicle's stepless speed.

\section{Composition and working principle of hydrostatic transmission vehicle}

The hydrodynamic drive vehicle has four hydraulic pumps and 14 drive motors, using a fully driven approach. The working principle of the hydraulic drive system is shown in Figure 1, which includes the engine 1, the hydraulic pump 2, the fuel tank 3 , the cartridge valve 4 , the electromagnetic directional valve 5 , the relief valve 6 , the hydraulic motor 7, Wheel reducer 8 and tire 9.

The engine 1 is connected to the hydraulic pump 2 to drive the hydraulic pump to rotate, to supply the

\footnotetext{
${ }^{\mathrm{a}}$ Corresponding author: zhaoliangslg@163.com
}

hydraulic oil to the travel drive system, to drive the hydraulic motor 7 via the cartridge valve 4 , to rotate the wheel 9 by the wheel speed reducer 8 , The solenoid valve 5 controls the cartridge valve 4 to change the flow of the hydraulic oil so that the vehicle is advanced or retreated. The constant power control of the hydraulic pump with the pressure cut-off is used, and the displacement of the hydraulic pump varies depending on the rotational speed of the engine 1 . The hydraulic motor 7 is controlled by the electrical ratio, and the displacement of the hydraulic motor is controlled by the magnitude of the current of the given hydraulic motor coil.

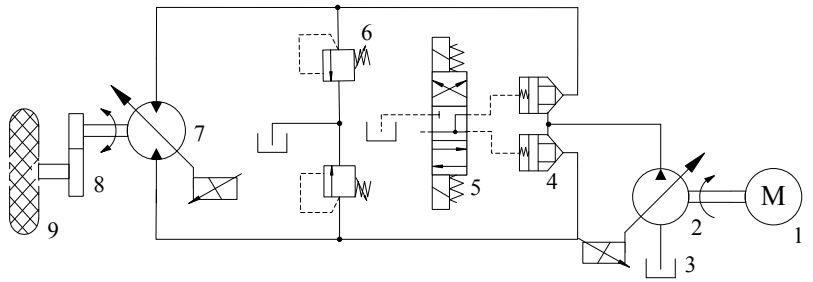

Figure 1. Schematic diagram of single-sided driving hydraulic system

\section{Matching and control of engine and hydraulic pump}

In order to make the engine in the vehicle running in the process of working in the best state, according to the external characteristics of the engine to control the displacement of the hydraulic pump, when the engine load rate of $90 \%$, the engine fuel consumption is the best, so according to the engine The relationship between the 
engine output torque $\mathrm{Me}$ and the speed $\mathrm{n}$ is obtained by the least squares method: ${ }^{3,4}$

$$
M_{e}=M(n)
$$

Where $M(n)$ is the engine output torque at different speeds.

The external characteristics of the engine are shown in Figure 2, where $n_{1}$ is the idle speed of the engine; $n_{2}$ is the engine maximum output torque when the speed; $n_{\mathbf{3}}$ is the engine maximum output torque when the speed.

Considering the economy of the engine, the maximum displacement control of the hydraulic pump at each speed is: ${ }^{5}$

$$
q_{b}=\frac{0.9 M_{e} 2 \pi}{\Delta p}
$$

Where $q_{b}$ is the displacement of the hydraulic pump; $\Delta p$ is the pressure difference between the outlet and inlet of the hydraulic pump.The displacement control curve of the hydraulic pump is controlled along the curve $\mathbf{1}^{\prime}-\mathbf{2}-\mathbf{3}$ in Figure2.

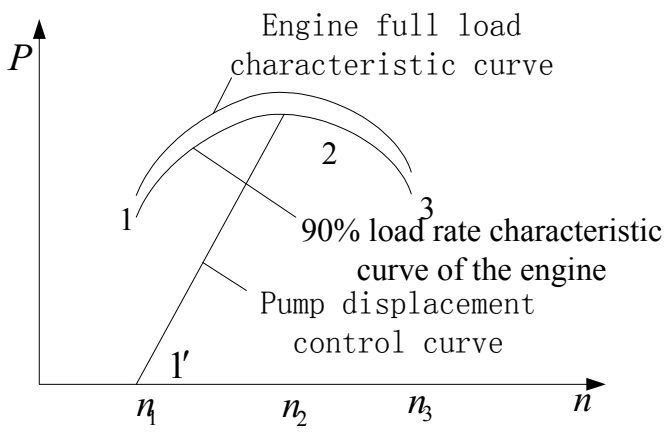

Figure 2. Maximum displacement control curve for hydraulic pumps

\section{Calculation of vehicle driving force}

Driving process, the chassis to provide the driving force and the resistance should be maintained in balance, the vehicle's driving equation is:

$$
F_{t}=F_{f}+F_{w}+F_{i}+F_{j}
$$

Where $F_{t}$ is the driving force; $F_{f}$ is the rolling resistance, $F_{f}=M g f ; F_{w}$ is the wind resistance, $F_{w}=\frac{C_{D} A v}{\mathbf{2 1 . 1 5}}, C_{D}$ is the wind resistance coefficient, $A$ is the windward area of the vehicle, $v$ is the speed of the vehicle; $F_{i}$ is the ramp resistance, $F_{i}=M g \sin \alpha$; $F_{j}$ is accelerated resistance.

\subsection{Driving force of maximum speed}

When the vehicle is traveling at the highest speed on the ground, The climb gradient $\alpha=\mathbf{0}$, so the ramp resistance $F_{i}=M g \sin \alpha=\mathbf{0} \quad$, The acceleration resistance $F_{j}=\mathbf{0}$. According to the design of the maximum speed, the wheel needs the driving force:

$$
F_{t}=F_{f}+F_{w}
$$

Given the parameters of the vehicle, you can determine the drive force required for the wheel,

Where $M=84000 \mathrm{~kg}, C_{D}=0.8$, $f=\mathbf{0 . 0 1 2}, A=5.5 \mathrm{~m}^{2}, v=\mathbf{8 0} \mathrm{km} / \mathrm{h}$, the driving

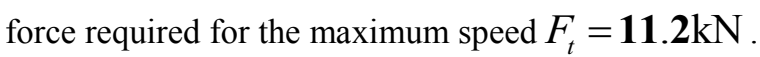

\subsection{Maximum driving force when climbing}

When the vehicle is running on the maximum slope, the speed of travel is relatively low, can ignore the wind resistance, driving resistance only rolling resistance and ramp resistance, which:

$$
F_{t}=F_{f}+F_{i}
$$

According to $30 \%$ of the gradient, $\alpha=\arctan (\mathbf{0 . 3})=16.67^{\circ}$, the driving force required for

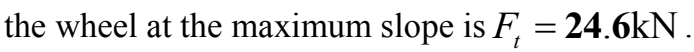

\section{Parameter design of hydraulic Motor}

In the hydraulic motor selection calculation, to make the hydraulic motor to meet the hydraulic drive vehicle power requirements and the maximum speed requirements, and can zero displacement. The vehicle has the greatest running resistance at the maximum slope, and the speed of the hydraulic motor is required to meet the requirements of the running speed when traveling on the ground.

The driving torque of the hydrostatic transmission vehicle:

$$
T_{m}=\frac{F_{t} R_{r}}{n i_{g} \eta_{g}}
$$

Where $n$ is the number of drive wheels when driving, $i_{g}$ is the gear ratio of the wheel reducer, $\eta_{g}$ is the mechanical efficiency of the gear unit, $R_{r}$ is the rolling radius of the wheel.

The hydraulic motor is also required to meet the maximum speed of the vehicle design, The maximum 
speed of the design is $v_{\max }=\mathbf{8 0} \mathrm{km} / h$. Calculate the maximum speed of the hydraulic motor according to the design maximum speed..

$$
n_{m \max }=\frac{v_{\max } \mathbf{6 0}}{2 \pi R_{r} \mathbf{3 . 6}} i_{g}
$$

Where $n_{m \max }$ is the maximum speed of the hydraulic motor, $i_{g}$ is the wheel reducer gear ratio, $R_{r}$ is the wheel rolling radius.

\section{System work pressure calculation}

The working pressure of the hydraulic motor is determined by the running resistance of the hydrostatic transmission vehicle. The working pressure is calculated as:

$$
\Delta P_{m}=\frac{100 T_{m}}{1.59 q_{m} \eta_{m h}}
$$

Where $\Delta P_{m}$ is the working pressure at both ends of the hydraulic motor, $T_{m}$ is the torque on the hydraulic motor, $q_{m}$ is the displacement of the hydraulic motor, $\eta_{m h}$ is the mechanical efficiency of the hydraulic motor.

\section{Parameter design of hydraulic pump}

Hydraulic pump in the calculation of parameters to meet the system's working pressure and hydraulic motor hydraulic flow requirements. Hydrostatic transmission vehicles in the ground to high speed, the hydraulic motor displacement to take $50 \mathrm{ml} / \mathrm{r}$, according to the number of drive the number of systems required flow:

$$
Q_{\max }=n_{\max } q_{m} n
$$

Where $n_{m \max }$ is the maximum speed of the hydraulic motor, $q_{m}$ is the displacement of the hydraulic motor, $n$ is the number of drive wheels.

Calculate the displacement of the hydraulic pump based on the maximum flow required by the system and the speed of the engine.

$$
q_{b}=\frac{Q_{\max }}{n_{b} n_{p}}
$$

Where $n_{b}$ is the number of hydraulic pumps, $n_{p}$ is the maximum speed at which the engine is operating.

\section{Braking system parameter calculation}

\subsection{Braking performance calculation}

Braking operation, close the motor control valve, while maximizing the motor displacement, the use of the relief valve motor outlet pressure to slow down the motor, in order to achieve braking. The maximum pressure of the entire walking hydraulic system is the system relief valve settings.

The maximum hydraulic resistance moment:

$$
M_{b r}=1.59 \frac{P_{b r} P_{m \max }}{10} i_{g} n
$$

Where $P_{b r}$ is the brake relief pressure, the minimum is set to $20 \mathrm{MPa}$, the maximum is $35 \mathrm{MPa}$, determined by the brake pedal position, $i_{g}$ is the wheel reducer gear ratio.

The maximum brake deceleration is:

$$
a_{b r}=\frac{M_{b r}}{R_{r} M}
$$

The full load brake the shortest distance:

$$
s=\frac{v^{2}}{2 a_{b r}}+v\left(t_{a}+\mathbf{0 . 5} t_{b}\right)
$$

Where $v$ is the full speed, determined according to the current speed, $t_{a}$ is the braking system reaction time, refers to the brake pedal when braking to overcome the free travel, hydraulic oil pressure transmission and other time required, the general hydraulic brake system reaction time is $0.015-0.03 \mathrm{~s}$, taken here $0.02 \mathrm{~s}$. $t_{b}$ is deceleration deceleration time, the general hydraulic brake system is $0.15-0.3 \mathrm{~s}$, taken here $0.3 \mathrm{~s}$.

\subsection{Braking performance calculation}

According to the design requirements, the parking slope is $30 \%$, about $16.7^{\circ}$ slope.

The parking brake torque to be provided by the wheel reducer is:

$$
T_{t}=\frac{M g \sin \alpha R_{s}}{n i_{g}}
$$

Where $M$ is the vehicle quality, $R_{s}$ is the wheel's static radius.

\section{Engineering case analysis}

According to the vehicle driving conditions, multiaxis hydrostatic transmission vehicle driving system hydraulic pump selection Rexroth A11VO190 variable pump, hydraulic motor selection A6VM200 variable motor, A11VO190 variable pump nominal pressure of 350 bar, A6VM200 variable motor nominal pressure of 400bar According to (6) and (8) to calculate the hydraulic motor working pressure range of $174 \mathrm{bar}$. 
318bar, can make the system pressure to get a better match. According to (11),(12) and (13), the running braking torque of the vehicle can be obtained as $M_{b r}=210 \mathrm{kN} \cdot \mathrm{m}$, the maximum running braking deceleration of the vehicle can be obtained as $a_{b r}=3.77 m / s^{2} ;$ According to (14), the parking brake torque of the vehicle can be obtained as $T_{t}=795 \mathrm{~N} \cdot \mathrm{m}$

\section{Conclusion}

In this paper, the parameter matching of driving system of multi-axis hydrostatic transmission vehicle is researched. The parameter matching of running drive system, driving resistance, parameters calculation of hydraulic motor and hydraulic pump, and running brake system are analyzed. The parameter calculation of the hydraulic drive system is explained, which provides the basis for the parameter calculation of the hydrostatic drive vehicle.

\section{Acknowledgement}

The article was supported by Special research project of Shaanxi Provincial Department of Education (Grant No. 14JK1134 ),Shaanxi Province Natural Science Basic Research Project(Grant No. 2017JM5087 ), Natural Science Project of Shaanxi Provincial Science and Technology Department (Grant No. 2016JM5039).

\section{References}

1. YAO Huaixin. "Construction machinery chassis and its hydraulic drive theory", China Communications Press, 2002

2. YAO Huaixin. "Rationale and Data Matching for Control of Vehicle's Hydraulic Driving Systems.ChinaJournal of Highway and Transport", 2002, 15(3):115-118

3. WANG Yongqi, SHAN Xinzhou. "Parameter matching and controlling of static hydraulic driving device in bulldozers". Construction mechanization, 2003 (10): :34-53

4. Wang Xin, Zhang Chao, Yi Xiaogang." Research on Power - Load Adaptive Method for Full Hydraulic Leveling Machine", 2007,(38)

5. LONG Shuigen, YANG Xilong, REN Shijie. "Matc Parameters Calculation and Matching in the Traveling Driven System of Full Hydraulic Bulldozer", Hydraulic and pneumatic, 2007(12) :97100

6. Zuo Delong, Yin Wenqing, Hu Fei . "Matching and Simulation of Engine and Hydrostatic Transmission", Mechanical transmission, 2010,(34)
7. ZHAO Liang. "Matching and control study on driving system of full hydraulically-driven chassis",Journal of China Construction Machinery Corporation, 2013, (6)

8. LI Xiang sheng, CHANG Siqin. "Matching Efficiency of Power Source System for Vehicle with Energy Accumulation Hydrostatic Drive", China Journal of Highway and Transport,2007 , 20(1) :118-122

9. Yi Xiaogang, Jiao Shengjie, Liu Zhengfu.” Research on Key Technical Parameters of Full Hydraulic Bulldozer" China Journal of Highway and Transport,2004(2)

10. LI Xiang sheng, CHANG Si qin."Design of the Power SourceSystem for the Vehicle of HydrostaticTransmission System "Journal of Nanjing Forestry University(Natural Sciences Edition,,2005,29(4) :65-68 